\title{
The Association of Compact Groups of Galaxies with Large-scale Structures
}

\author{
Heinz Andernach and Roger Coziol \\ Departamento de Astronomía, Universidad de Guanajuato, Mexico \\ heinz@astro.ugto.mx, rcoziol@astro.ugto.mx
}

Summary. We use various samples of compact groups (CGs) to examine the types of association CGs have with rich and poor clusters of galaxies at low $(z \simeq 0.04)$ and intermediate $(z \simeq 0.1)$ redshifts. We find that $\sim 10-20 \%$ of CGs are associated with rich clusters and a much larger fraction with poorer clusters or loose groups. Considering the incompleteness of catalogs of poorer systems at intermediate redshift, our result is consistent with all CGs at intermediate redshift being associated with larger-scale systems. The richness of the clusters associated with CGs significantly increases from $z \simeq 0.04$ to $z \simeq 0.1$, while their Bautz-Morgan type changes from early to late type for the same range in $z$. Neither trend is compatible with a selection effect in the cluster catalogs used. We find earlier morphological types of galaxies to be more frequent in CGs associated with larger-scale structures, compared to those in CGs not associated to such structures. We consider this as new evidence that CGs are part of the large-scale structure formation process and that they may play an important role in the evolution of galaxies in these structures.

\section{Motivation and Method}

Although groups of galaxies form the principal environment of most galaxies in the Universe, their origin and evolution are still poorly understood. Theoretically, the resolution of numerical simulations is still insufficient to understand the formation of such small-scale structures. Observationally, the intrinsic low richness of groups makes them more difficult to detect and study than richer systems like cluster of galaxies, especially at intermediate and high redshift.

Within the Hierarchical Structure Formation paradigm (based on CDM models) we would like to address questions like e.g. "When do groups form and what IMF do they follow?", or "Assuming galaxy formation is biased, should we expect a sort of downsizing effect for groups, i.e. high-mass groups at high z forming before lower-mass ones at lower z?" What is the role of groups in the formation of large-scale structures? If groups were merging together to form larger-scale structures, how long can we expect them to retain their dynamical characteristics and be distinguished from the larger, more massive structure? Finally, following the merging scenario, what is the importance of groups in the evolution of galaxies located in large-scale structures? 
Here we concentrate on Compact Groups of galaxies (CGs). The advantage of CGs over other groups is that they reach galaxy densities comparable to those of clusters, and are consequently easier to identify in the sky, even at moderate redshift. From their relation with larger-scale structures 9, [12, 10, 2] it seems clear also that CGs form part of the large-scale structure formation process. Finally, it is expected that such compact configurations should have some distinctive and recognizable impact on the evolution of their galaxy members (see e.g. Plauchu Frayn et al., these proceedings). A more detailed study of the relation of CGs with large-scale structures may yield new insights on some of the above questions.

To examine the relation CGs have with large-scale structures we compiled a list of possible associations of CGs with rich and poor clusters of galaxies and with loose groups of galaxies. The CG samples we use are the HCGs [5], the SCGs [6], the SDSSCGs [8], and the PCGs [3. Note that in our previous analysis [2] only the preliminary PCG sample by [7] was available. As samples of rich clusters we use the Abell catalog 1, and for poorer clusters and loose groups we take the NSC 4] and USGC [1] samples, respectively. The association method we use is described in detail in [2].

Based on the position of the groups relative to the center of the associated larger-scale structure and on a comparison of the apparent magnitude of galaxies in both structures we identified three types of such associations: ML or AML indicate that the galaxies in the CG are either the "Most Luminous" or "Among the Most Luminous" ones of the associated larger-scale structure, and SS if the group forms some sort of substructure at the periphery of a larger system. Examples of each association class can be found in [2].

\section{Results}

\subsection{Associations of CGs with rich clusters}

The associations of CGs with Abell clusters are described in Table 1 Columns are the CG sample name, total number of CGs in each sample (note that on the basis of our optical inspection of all 459 published PCGs we discarded 52 , mostly for stellar contamination), the number of CGs associated with an Abell cluster, the percentage of associated CGs, their mean redshift, the number distribution of associated CGs among the different association types,

and the Abell richness classes as well as the Bautz-Morgan (BM) types of the associated clusters. Interesting differences are observed, which can easily be related to the different group selection methods. Eye selection of the CG candidates, like HCGs and visual inspection of the otherwise automatically selected groups like the SDSSCGs, seem to discard most associations of CGs with larger-scale structures: $83 \%$ of HCGs and $69 \%$ of the SDSSCG are SS, while $57 \%$ are ML type in the SCGs and $72 \%$ are AML in the PCGs. This difference in the selection method may also explain why we found only 
one coincidence of a SDSSCG with a PCG. Based on the surface density of SDSSCGs and PCGs and their region of overlap, one would expect about 10 coincidences if the two selection methods were identical.

Table 1. Associations of CGs with Abell clusters

\begin{tabular}{|c|c|c|c|c|c|c|c|c|c|c|c|c|c|c|}
\hline \multirow{3}{*}{$\begin{array}{l}\mathrm{CG} \\
\mathrm{HCG}\end{array}$} & \multirow[t]{2}{*}{$\mathrm{N}_{\mathrm{CG}}^{\text {tot }}$} & \multirow[t]{2}{*}{$N_{\text {ass }}$} & \multirow[t]{2}{*}{$\%$} & \multirow[t]{2}{*}{$\langle z\rangle$} & \multirow{2}{*}{\multicolumn{3}{|c|}{$\begin{array}{l}\text { Ass. type } \\
\text { SS ML AML }\end{array}$}} & \multicolumn{4}{|c|}{ Richness } & \multirow{2}{*}{\multicolumn{3}{|c|}{$\begin{array}{c}\text { BM type } \\
\text { undef. early late }\end{array}$}} \\
\hline & & & & & & & & 0 & 1 & 2 & 3 & & & \\
\hline & 100 & 6 & 6 & .04 & 5 & 1 & $\cdots$ & 5 & $\ldots$ & $\ldots$ & 1 & $\ldots$ & 2 & 3 \\
\hline SCG & 121 & 21 & 17 & .04 & 9 & 12 & $\ldots$ & 18 & 2 & 1 & $\ldots$ & 2 & 14 & 5 \\
\hline PCG & 407 & 71 & 17 & .12 & 20 & $\ldots$ & 51 & 23 & 34 & 12 & 3 & 12 & 5 & 54 \\
\hline SDSSCG & 177 & 16 & 9 & .12 & 11 & $\ldots$ & 5 & 5 & 9 & 1 & 1 & 4 & $\ldots$ & 12 \\
\hline
\end{tabular}

Despite the difference in group selection techniques the CG-cluster association rates seem quite similar at low $(z=0.04)$ and intermediate $(z=0.12)$ redshifts.

Other differences observed are more difficult to explain. We note for example that the richness of the associated clusters rises with redshift: the fraction of $\mathrm{R}=1$ cluster rises from $14 \%$ in the SCGs to $69 \%$ in the PCGs and SDSSCGs. The average BM type also seems to change with redshift: passing from $74 \%$ early types (I, I-II, II) in the SCGs to $92 \%$ late types (II-III, III) in the PCG and $100 \%$ late types in the SDSSCG.

We already observed these differences in our first examination of these samples [2]. The fact that we observe the same phenomenon using the complete (and revised) sample of PCGs and the SDSSCG sample, confirms that these differences occur in relation with the increase in redshift. In [2] we verified that these differences cannot be due to an incompleteness in richness of the Abell/ACO sample at intermediate redshift, nor to some sort of luminosity bias affecting clusters with different BM types. These differences are consistent with an increase of the mass of the associated structures at increasing redshift.

\subsection{Associations of CGs with poorer structures}

The associations of CGs with large-scale structures poorer than Abell clusters are described in Table 2 Columns are the CG sample name, the sample name of the associated structure, the number and percentage of CGs associated with that structure, the mean redshift of the CG-associated structures, their distribution among the three association types defined earlier, and the average richness of the associated structure.

Comparing with Table 1 we see that CGs are much more frequently associated with poorer than with richer structure. This increase in the number of associations is significant: a factor of 2 to 6 for the SCG and HCG at low 
$z$, and a factor of 3 to 4 for the SDSSCG and PCG at intermediate $z$. Note that the severe incompleteness of the NSC in low-richness structures (cf. 4]) implies that at higher redshifts almost all CGs may be part of a larger-scale system.

In Table 2 we also observe a significant increase in the number of MLand AML-type association which indicates that CGs may generally form important "substructures" in these poorer, larger-scale systems.

Table 2. Associations of CGs with poorer structures

\begin{tabular}{|c|c|c|c|c|c|c|c|c|}
\hline \multirow[t]{2}{*}{$\mathrm{CG}$} & \multirow[t]{2}{*}{ LSS } & \multirow[t]{2}{*}{$\mathrm{N}_{\mathrm{CG}}^{\text {assoc }}$} & \multirow[t]{2}{*}{$\%$} & \multirow[t]{2}{*}{$\langle z\rangle$} & \multicolumn{3}{|c|}{ Assoc. type } & \multirow[t]{2}{*}{$\left\langle\mathrm{N}_{\text {gal }}\right\rangle$} \\
\hline & & & & & & ML & AML & \\
\hline $\mathrm{HCG}$ & USGC & 37 & 37 & 0.02 & 2 & 30 & 5 & 6 \\
\hline $\mathrm{SCG}$ & USGC & 33 & 27 & 0.02 & 8 & 22 & 3 & 8 \\
\hline PCG & NSC & 195 & 62 & 0.13 & 55 & 10 & 130 & 35 \\
\hline SDSSCG & NSC & 21 & 25 & 0.13 & 5 & $\ldots$ & 14 & 40 \\
\hline
\end{tabular}

\subsection{Variation of galaxy morphologies with CG environment}

In Table 3 we compare the number of galaxies of early (E-S0-S0/a), intermediate (Sa-Sab-Sb) and late (Sbc and later) morphological type in CGs located in different large-scale environments. For both HCGs and SCGs, distinguishing whether they are or not associated with clusters or loose groups (LGs), we list the number and percentages of galaxies of different morphological types. $\mathrm{N}_{\text {gal }}$ is the number of galaxies for which we could find a morphological type in NED (nedwww.ipac.caltech.edu). As expected, we find a significant increase of earlier morphological types in CGs associated with large-scale structures. This result suggests that in general the associations we found are physically real.

Table 3. Distribution of galaxy morphologies as a function of CG environment

\begin{tabular}{|c|c|c|c|c|c|c|c|c|c|}
\hline \multirow{2}{*}{$\begin{array}{l}\text { Morphological } \\
\text { type }\end{array}$} & \multicolumn{2}{|c|}{$\begin{array}{c}\text { HCG } \\
\text { out of } \mathrm{cl} .\end{array}$} & \multicolumn{2}{|c|}{$\begin{array}{l}\mathrm{HCG} \\
\text { in LGs }\end{array}$} & \multicolumn{2}{|c|}{$\begin{array}{c}\mathrm{HCG} \\
\text { in cl. }\end{array}$} & \multicolumn{2}{|c|}{$\begin{array}{c}\text { SCG } \\
\text { out of } \mathrm{cl} .\end{array}$} & \multirow{2}{*}{$\begin{array}{c}\mathrm{SCG} \\
\text { in cl. } \\
\mathrm{N}_{\text {gal }} \%\end{array}$} \\
\hline & $\mathrm{N}_{\text {gal }}$ & $\%$ & $\mathrm{~N}_{\text {gal }}$ & $\%$ & $\mathrm{~N}_{\text {gal }}$ & $\%$ & $\mathrm{~N}_{\text {gal }}$ & $\%$ & \\
\hline Early & 142 & 54.4 & 73 & 46.8 & 17 & 65.4 & 72 & 45.0 & 2264.7 \\
\hline Intermediate & 33 & 12.6 & 28 & 17.9 & 2 & 7.7 & 49 & 30.6 & 720.6 \\
\hline Late & 86 & 33.0 & 55 & 35.3 & 7 & 26.9 & 39 & 24.4 & $\begin{array}{ll}5 & 14.7\end{array}$ \\
\hline
\end{tabular}

On the other hand, it is also quite interesting to note how rich in earlytype galaxies the isolated CGs already are. Note that there seems to be 
no difference between isolated CGs and those associated with loose groups (LG). These results suggest that compact configurations like CGs have a great influence on galaxy evolution. This may be independent of the environment or it may depend on some threshold mass of the larger-scale structure with which the CG is associated.

\section{Conclusions}

Despite the obvious biases introduced by the different selection methods, the high number of associations of CGs with larger-scale structures encountered in our analysis indicates that these systems must form naturally during the large-scale structure formation process. Both, the increase of mass of the associated structures at intermediate redshift, and the variation of galaxy morphologies with CG environment, are consistent with biased galaxy formation, which implies some sort of downsizing effect for groups: more massive groups form before less massive ones. Compact groups may affect galaxy evolution independently of environment, and/or the CG environment may affect galaxies more strongly above some threshold mass of the structures in which the groups form.

\section{References}

1. Abell G.O., Corwin Jr. H.G., Olowin R.P., ApJS, 70, 1 (1989)

2. Andernach H., Coziol R., in Nearby Large-Scale Structures and the Zone of Avoidance, ASP Conf. Ser., 239, eds. A.P. Fairall \& P.A. Woudt, p. 67 (2005)

3. de Carvalho R.R., Gonçalves T.S., Iovino A., et al.: AJ, 130, 425 (2005)

4. Gal R.R., de Carvalho R.R., Lopes P.A.A., et al.: AJ, 125, 2064 (2003)

5. Hickson P., Mendes de Oliveira C., Huchra J.P., et al., ApJ, 399, 353 (1992)

6. Iovino A., AJ, 124, 2471 (2002)

7. Iovino A., de Carvalho R.R., Gal R.R., et al.: AJ, 125, 1660 (2003)

8. Lee B.C., Allam S.S., Tucker D.L., et al.: AJ, 127, 1811 (2004)

9. Mamon G.A., A\&A, 219, 98 (1989)

10. Ramella M., Diaferio A., Geller M.J., Huchra J.P., AJ, 107, 1623

11. Ramella M., Geller M.J., Pisani A., da Costa L.N., AJ, 123, 2976 (2002)

12. Rood H.J., Struble M.F.: PASP, 106, 413 (1994) 